\title{
Impacto de la capacitación en negociación en su aplicación práctica durante su gestión laboral
}

\author{
Oscar Reynaldo Talavera Velásquez ${ }^{1}$ \\ Escuela de Postgrado, Universidad Peruana de Ciencias Aplicadas (UPC, Lima, Perú)
}

\section{RESUMEN}

Este artículo busca analizar la literatura existente sobre el impacto de la capacitación en negociación en el desempeño de profesionales. Se recolectaron artículos de las bases de datos de Web of Science y se analizaron para identificar los aspectos donde una investigación podría contribuir al conocimiento actualmente del tema.

\section{PALABRAS CLAVE}

Capacitación; negociación; desempeño laboral

Impact of training in negotiation on its practical application in management

\section{ABSTRACT}

This article analyzes the existing literature on the impact of training in professional development. Articles from the Web of Science database were analyzed to identify aspects where additional research would contributed to current knowledge on the subject.

\section{KEYWORDS}

${ }^{1}$ Director del Área Académica de Ingeniería. Correo: oscar.talavera@upc.edu.pe.

Talavera Velásquez, O. R. (2015). Análisis del impacto de la capacitación en negociación en su aplicación práctica durante su gestión laboral. Sinergia e Innovación, 3(1), 121-133.

Fecha de recepción: 01/04/15

Fecha de aceptación: 30/04/15 
Training, negotiation, professional, performance 


\section{Introducción}

El mundo actual cuenta con una transferencia cada vez más rápida de información y tecnología entre los diferentes países del mundo; los profesionales que realizan transacciones, acuerdos, contratos y demás actividades necesitan contar con herramientas que les permitan alcanzar sus objetivos a la hora de realizar una negociación.

Los cambios en los puestos de trabajo, tales como el aumento de la tecnología y la influencia de los mercados internacionales en la competencia por mercados, han llevado a las organizaciones a depender cada vez más de su capacidad de capacitar a sus empleados en forma permanente para mantener los niveles necesarios de conocimiento, habilidad y rendimiento como una manera de obtener una ventaja competitiva frente a sus competidores. Es importante que los estudiantes aprendan los conocimientos y habilidades necesarias y que este aprendizaje se transfiera con éxito a las empresas (Weissbein, Huang, Ford \& Schmidt, 2011).

El supuesto implícito que subyace a la formación en gestión es que una vez presentada y entendida, los conocimientos de gestión se puede aplicar a los problemas futuros que los gerentes pueden enfrentar (Thompson, Gentner \&Loewenstein, 2000). Por otro lado, la negociación requiere de habilidades gerenciales fundamentales pero complejas (Zerres, Hüffmeier, Freund, Backhaus \&Hertel, 2013).

Muchas organizaciones tienen centros de formación para mejorar las competencias de los empleados. Los programas de formación abarcan una amplia gama de temas, tales como capacidades técnicas, de gestión y administrativas. Un ejemplo se encuentra en el trabajo de Yeganeh \& Su (2008), en el cual se explica que el concepto de gestión de recursos humanos es esencialmente americana pero hoy en día se puede considerar un concepto internacional que se puede aplicar a otros países (Brewster, 1995). Ferris, Rosen, \& Barnum (1995) dieron una definición de gestión de recursos humanos, formulando este concepto de la siguiente manera: "Gestión de recursos humanos es la ciencia y la práctica que tienen que ver con la naturaleza de la relación de trabajo y todas las decisiones, acciones y temas que se relacionan con esa relación", es decir, de mejorar las relaciones de trabajo, el ambiente laboral, y la toma de decisiones. Esto evidencia que en todas las partes del mundo este tipo de capacitación es un instrumento necesario para mejorar el desempeño profesional, dentro de estas capacitaciones se cubren el desarrollo de habilidades como la negociación. 
Por tanto, el impacto del entrenamiento en negociación es reconocido en distintos países, por lo que se necesita reforzar las herramientas de negociación en los profesionales, los cuales les permitan ser competitivos en la arena nacional y la internacional (Gillespie, Thompson, Loewenstein \& Gentner, 1999).

Según Ogilvie \&Simms (2009), hay un resurgimiento de interés en la investigación sobre la negociación; sin embargo, de acuerdo a Gillespie, Thompson, Loewenstein \& Gentner (1999), después de dos décadas de un crecimiento espectacular en la investigación de negociación, no hay una investigación que brinde una visión entre la pedagogía y la práctica.

\section{Planteamiento del problema}

Tal como lo explica Wheeler (2004), en ventas, el inicio de las negociaciones recibe escasa atención en la literatura sobre negociación. Gran parte de lo que está escrito tiende a ser en torno a cuestiones tácticas, sin embargo, nada está dicho acerca de cómo se maneja la presión de otra persona para llegar a un precio de venta o para atreverse a hacer una demanda extravagante. Las herramientas y la preparación en temas de negociación deben permitir al profesional adaptarse a distintos escenarios, a distintas barreras culturales, económicas, sociales; y debe facilitar una relación a largo plazo con su contraparte.

Por otro lado, según Román \& Ruiz (2003), un profesional necesita capacitación que le permita interactuar de manera exitosa con su entorno, ya sean sus superiores, las personas que están bajo su mando, sus compañeros, y de manera más notoria los profesionales de otras instituciones.

El objetivo es preparar estudiantes y administradores a resolver problemas del mundo real con el aprendizaje en el aula. La evidencia empírica muestra la poca capacidad de las personas para aprender con el ejemplo y aplicar el conocimiento a la hora de resolver un problema en un contexto diferente. Mientras no se tenga claro si la capacitación en negociación mejora la gestión de las personas que la reciben, no se podrá seguir alguna tendencia de los modelos a cursar o los métodos a usar en la capacitación, sólo tendremos análisis de laboratorio (Loewenstein, Thompson \&Gentner, 2003).

En este proceso de transferencia, lo que es más útil es el razonamiento; a menudo no se recuerda en última instancia lo que es más valioso para la solución de un problema (Forbus, Gentner \&Law, 
1995). Los resultados de varias investigaciones señalan una disociación entre lo que es más accesible a la memoria y la aplicación a la práctica (Loewenstein, Thompson \&Gentner, 2003).

En conclusión, las negociaciones se encuentran en un amplia variedad de entornos de negocios (Nadler, Thompson \& Van Boven, 2003), por lo tanto, vale la pena aprender los principios de negociación de manera que maximicen de que se utilicen en todos los contextos (Forbus, Gentner \&Law, 1995). Por ello, es importante medir el impacto de la capacitación en negociación para mostrar que hay un impacto de esta capacitación en la gestión futura de los capacitados.

Las habilidades personales son complementarias a los conocimientos y habilidades técnicas. Los conocimientos pueden ser estudiados y aprendidos en cualquier momento, pero son las habilidades personales las que hacen destacar a las personas. En la medida que un profesional avanza en su carrera, su desempeño depende cada vez más de sus habilidades personales que de sus conocimientos o habilidades técnicas

Este artículo busca analizar la literatura existente sobre el impacto de la capacitación en negociación en el desempeño de profesionales. La búsqueda de datos se realizó en junio del 2014. La estrategia de búsqueda consistió en buscar artículos con los siguientes términos y truncamientos (sin comillas): "negociation", "negotiate", "nego*", "training", "train*”, "impacto" e "impact". Se consultaron las siguientes bases y años del Web of Science:

- Century of Social Science del Web of Science, base de más de 300 revistas de investigación en números desde (1900-presente).

- Science Citation Index Expanded (1900-presente).

- Arts \& Humanities Citation Index (1975-presente)

- Conference Proceedings Citation Index - Science (1990-presente)

- Conference Proceedings Citation Index - Social Science and Humanities (1990-presente)

- Book Citation Index - Science (2005-presente)

- Book Citation Index - Social Science and Humanities (2005-presente)

Se filtraron los resultados por las siguientes temáticas: "business", "management" y "business finance" y resultaron 132 registros. 


\section{Análisis de datos}

Desde hace más de veinticinco años la habilidad de la negociación ha sido ampliamente reconocida tanto como un tema de investigación y como un elemento esencial de uso frecuente (Movius, 2008).Movius (2008) señala que la mayoría de estudios sobre entrenamiento en negociación se han realizado en laboratorios o mediante negociaciones simuladas analizando la formación didáctica, analítica y observacional; pero pocos han observado el impacto a largo plazo en el comportamiento por la capacitación en negociación.

Ahora, según Román \& Ruiz (2003), si bien la globalización y sus efectos han conectado a empresas en todo el mundo este proceso no conlleva a la pérdida de las costumbres ni las tradiciones culturales de cada región o país en su totalidad. Por lo tanto, la enseñanza debe tener en cuenta no sólo los factores sociales y económicos, sino también los culturales es por esto que ésta investigación que se hace a nivel global, tiene que tomar en cuenta la realidad, ubicación y cultura del país en la que la estamos realizando. El presente estudio tendría un enfoque que debe visualizarse bajo realidades parecidas al país.

La negociación es generalmente considerada como una de las habilidades esenciales necesarias para la eficacia de la organización y el éxito (Volkema, 2009). Las personas en todo tipo de organizaciones y en todos los niveles organizativos negocian a diario; dada la importancia de este atributo, muchas de las habilidades de negociación son empleadas en negociaciones personales y profesionales (Lewicki, Barry, \& Saunders, 2010).

El trabajo de Fisher, Ury \& Patton (2011) comienza diciendo que cada vez hay más ocasiones que requieren una negociación, el conflicto es una industria en desarrollo. Todo el mundo quiere participar en las decisiones que le afectan y, por el contrario, cada vez son menos las personas que pueden llegar a aceptar decisiones que han sido tomados por otros. Ahora bien, la negociación necesita que las partes que participan tengan como objetivo el éxito de ambas. Las interacciones entre los seres humanos podrían volverse más efectivas, si es que se capacitarán en negociación. Tal como lo menciona este trabajo, usualmente nos enfocamos en posiciones a la hora de negociar, lo que no permite encontrar resultados óptimos ni siquiera para nosotros mismos. Uno de los retos más importantes en negociación son las relaciones interpersonales. Por tanto, el comportamiento humano no debe ser ajeno al negociador. 
La capacitación en negociación se inició en Cambridge University a inicios de 1980, inmediatamente en el 1981 en Harvard University se crea el programa de negociación liderado por Roger Fisher y William Ury, autores del libro Getting to Yes. En 1982, Howard Raiffa publica el libro The Art and Science of Negotiation, la cual investiga sobre el comportamiento de las personas basado en la racionalidad y la negociación como herramienta para evitar conflictos. En el año 1986 David Lax y James Sebenius en su libro Manager as Negotiator describen la necesidad de las conductas de las contrapartes (Tsay \& Bazerman, 2009).

Hay otros dos factores interesantes que señalan Román y Ruiz (2003). El primero es que la capacitación en negociación es un elemento necesario que toda empresa debe promover en su empresa o negocio, es decir, deben ser ellos quienes la fomenten. El segundo factor, tal como lo menciona Tsay \& Bazerman (2009), es que el entrenamiento en negociación ya no se enfoca necesariamente en la teoría de juegos; sino que ha desarrollado una estrategia en base a un análisis sobre los verdaderos agentes imperfectos como son las personas en la realidad. Por tanto, se enfoca en dar consejos a los negociadores que se encuentran en negociaciones con personas reales.

Tal como lo menciona Román \& Ruiz (2003), este entrenamiento permite estar mejor preparado para cerrar negociaciones ya que logra manejar conflictos entre culturas distintas. De esta manera, un vendedor que ha recibido entrenamiento puede superar las vallas culturales y lograr relaciones a largo plazo. Por tanto, mientras más habilidades de negociación tenga la persona, más posible es que su desarrollo en la negociación sea impecable.

¿Cuál es el significado de negociación?. Según Movius (2008), la negociación es un proceso en el que dos o más partes buscan un acuerdo sobre lo que cada uno dará y tomará de la otra. Por otro lado, Fisher, Ury \& Patton (2011) definen negociación como un método de doble vía, en donde se comparten intereses comunes y opuestos, buscando ventajas individuales y comunes que permitan llegar a un acuerdo. Otra definición la encontramos en Zerres, Hüffmeier, Freund, Backhaus \& Hertel (2013), en donde se define que la negociación es convencionalmente concebida como un conjunto de procesos de toma de decisiones interpersonal, proceso que implica la comunicación entre al menos dos partes con intereses divergentes para llegar a un acuerdo.

¿Por qué es importante la negociación? Tal como lo menciona Fisher, Ury \& Patton (2011), la negociación hasta hace poco era considerada como un arte, sin embargo, hoy se entiende como 
una técnica o conjunto de técnicas. Se ha pasado de un tratamiento intuitivo a un análisis sistemático del proceso. En el mundo empresarial, la negociación es una competencia que se le exige a todo profesional, ya que del buen accionar en este campo depende los resultados que se obtengan en la interacción con otros departamentos de la empresa, así como otros clientes y proveedores. Por tanto, se requiere preparación, resolución y flexibilidad.

¿Qué significa la negociación para un profesional? La negociación es importante debido a que una buena negociación, tal como mencionan Fisher, Ury \&Patton (2011), no significa llegar a cualquier acuerdo sino que significa llegar al mejor acuerdo posible. Es un intento que a través de un intercambio de puntos de vista y de argumentos buscan como fin producir un resultado acordado y aceptable para todas las partes implicadas.

Como mencionan Fisher, Ury \&Patton (2011), si bien no existe receta para convertir a un ejecutivo en un negociador perfecto, se lo puede convertir en uno más racional. Si queremos obtener un acuerdo beneficioso para nosotros, nuestro oponente deberá estar persuadido de que dicho acuerdo es también bueno para él. La persuasión es la consecuencia de la adhesión a que el otro ha sido invitado.

Esto nos permite alcanzar un objetivo para satisfacer ambas necesidades. Sólo existe negociación si hay esfuerzo por parte de los implicados para llegar a un acuerdo. Ambas partes deberán terminar la negociación con el sentimiento de haber ganado: eso es una verdadera negociación un juego de ganar-ganar, donde el sentimiento final es que se ha logrado el objetivo por parte de los intervinientes sean de una parte o de otra.

Spector (1997) se preguntó si los buenos negociadores nacen o se pueden formar mediante la capacitación en negociación, respondiéndose que sí hay personas que nacen con ciertas habilidades pero que también se pueden desarrollar buenos negociadores a través de la práctica soportados en herramientas y procesos; Gentner, Loewenstein \& Thompson (2003) también concluyen que estas habilidades pueden mejorar a través de la formación de negociación.

Herbst \& Schwarz (2011) destacan que se sabe muy poco del impacto que genera la capacitación en negociación sobre los estudiantes cuando estos lo llevan a la práctica. Si bien se han realizado estudios comparativos con estudiantes con capacitación y sin capacitación, se ha confirmado que los estudiantes con capacitación se desempeñan mejor, sin embargo, todo se hace en un medio simulado. Concluye que muchas preguntas en el campo de la negociación pueden ser probados 
de manera efectiva mediante el uso de estudiantes, dejando claro que estos resultados no debe interponerse en el camino a investigar con grupos de profesionales en entornos reales donde se desenvuelven. Terminan señalando que la investigación de la influencia de la experiencia en la capacitación en negociación está lejos de ser completa. La cuestión de la validez de esta investigación es demasiado importante como para dejarlo sin resolver.

Asimismo, muy pocos estudios se han publicado en relación a los efectos de los enfoques pedagógicos con el impacto en la negociación (Movius, 2008). Si bien hay estudios del impacto de negociación una vez terminada la capacitación, no hay estudios que muestran que esta capacitación trascienda en el largo plazo (Zerres, Hüffmeier, Freund, Backhaus \&Hertel, 2013).

Loewenstein, Thompson \& Gentner (2003) hicieron una investigación sobre la capacitación a ejecutivos mediante un día completo de seminario de formación en negociación, concluyendo que los ejecutivos manifestaron que habían aprendido nuevas habilidades pero a pesar de esto, no se logró obtener ninguna medida del impacto en la práctica profesional.

Igualmente Lewicki (1997), señaló que los ejecutivos de un taller de negociación reaccionaron bien a la formación; pero no se tiene información de un resultado específico.

Los investigadores en negociación hasta el momento han llegado a un consenso sobre el asunto, no hay pruebas concluyentes para probar o refutar la afirmación que la investigación llevada a cabo con estudiantes es de igual aplicabilidad general de la desarrollada por profesionales en negociaciones (Herbst\& Schwarz, 2011).

Asimismo, como señala Hofstede, Jonker \&Verwaart (2012), la negociación se diferencia en las diferentes culturas, los agentes negociadores son meticulosos al estructurar sus estrategias no dejando de tomar en cuenta las culturas nacionales

Según Ma (2007), las diferentes prácticas de los gestores de las empresas en negociación obligan a diferenciar los países de origen para identificar los arraigos culturales teniendo que adoptar estrategias diferenciadas

Las interacciones entre las empresas no pueden dejar de lado estas características y menos en una negociación. El éxito de la negociación es reflejo de las habilidades del profesional en adecuarse a la cultura de su contraparte (Román \& Ruiz, 2003). 
Por la misma razón, si las percepciones culturales de los agentes son distintas, es necesario que el entrenamiento en negociación en cada país sea diferente adecuándose a sus realidades. Es de esperar entonces que haya países que cuenten con entrenamientos donde el instructor esté más involucrado y sea más participativo, otros donde realmente tenga un trato más ceremonial (Román \& Ruiz, 2003). 


\section{Referencias}

Bazerman, M. H., \& Neale, M. A. (1993).La negociación racional: en un mundo irracional. Barcelona: Paidós.

Brewster, C. (1995). Towards a 'European' model of human resource management.Journal of International Business Studies, 1-21.

Ferris, G., Rosen, S. D., \& Barnum, D. T. (Eds.).(1995). Handbook of human resource management.Nueva York: Blackwell Publishing.

Fisher, R., Ury, W. L., \& Patton, B. (2011).Getting to Yes: negotiating agreement without giving in. Nueva York: Penguin.

Forbus, K. D., Gentner, D., \& Law, K. (1995). MAC/FAC: A Model of Similarity-Based Retrieval. Cognitive Science, 19(2), 141-205.

Gentner, D., Loewenstein, J., \& Thompson, L. (2003). Learning and transfer: a general role for analogical encoding. Journal of Educational Psychology, 95(2), 393.

Gillespie, J. J., Thompson, L. L., Loewenstein, J., \&Gentner, D. (1999).Lessons from analogical reasoning in the teaching of negotiation. Negotiation Journal, 15(4), 363-371.

Herbst, U., \& Schwarz, S. (2011). How valid is negotiation research based on student sample groups? New insights into a long-standing controversy. Negotiation Journal, 27(2), 147-170.

Hofstede, G. J., Jonker, C. M., \&Verwaart, T. (2012).Cultural differentiation of negotiating agents.Group Decision and Negotiation, 21(1), 79-98.

Lewicki, R. J. (1997). Teaching negotiation and dispute resolution in colleges of business: The state of the practice. Negotiation Journal, 13(3), 253-269.

Lewicki, R. J., Barry, B., \& Saunders, D. M. (2010). Essentials of negotiation. Boston: McGraw-Hill.

Loewenstein, J., Thompson, L., \& Gentner, D. (2003). Analogical learning in negotiation teams: Comparing cases promotes learning and transfer. Academy of Management Learning \& Education, 2(2), 119-127. 
Ma, Z. (2007). Conflict management styles as indicators of behavioral pattern in business negotiation: the impact of contextualism in two countries. International Journal of Conflict Management, 18(3), 260-279.

Movius, H. (2008), The Effectiveness of Negotiation Training. Negotiation Journal, 24: 509-531.

Nadler, J., Thompson, L., \&Boven, L. V. (2003).Learning negotiation skills: Four models of knowledge creation and transfer.Management Science, 49(4), 529-540.

Ogilvie, D., \& Simms, S. (2009). The Impact of Creativity Training on an Accounting Negotiation. Group Decision \& Negotiation, 18(1), 75-87.

Román, S \& Ruiz, S. (2003). A comparative analysis of sales training in Europe: Implications for international sales negotiations. International Marketing Review, 20(3), 304 - 327.

Spector, B. I. (1997). Analytical support to negotiations: An empirical assessment. Group Decision and Negotiation, 6(5), 421-436.

Thompson, L., Gentner, D., \&Loewenstein, J. (2000). Avoiding missed opportunities in managerial life: Analogical training more powerful than individual case training. Organizational Behavior and Human Decision Processes, 82(1), 60-75.

Tsay, C. J., \&Bazerman, M. H. (2009). A Decision-Making Perspective to Negotiation: A Review of the Past and a Look to the Future. Negotiation Journal, 25(4), 467-480.

Volkema, R. J. (2009). Why Dick and Jane don't ask: Getting past initiation barriers in negotiations. Business Horizons, 52(6), 595-604.

Weissbein, D. A., Huang, J. L., Ford, J. K., \& Schmidt, A. M. (2011). Influencing learning states to enhance trainee motivation and improve training transfer. Journal of Business and Psychology, 26(4), 423-435.

Wheeler, M. (2004). Anxious moments: Openings in negotiation. Negotiation Journal, 20(2), 153169.

Yeganeh, H \& Su, Z. (2008) An examination of human resource management practices in Iranian public sector. Personnel Review, 37(2). 203-221. 
Zerres, A., Hüffmeier, J., Freund, P. A., Backhaus, K., \&Hertel, G. (2013). Does it take two to tango? Longitudinal effects of unilateral and bilateral integrative negotiation training. Journal of AppliedPsychology, 98(3), 478. 